\title{
The impact of biomaterials on pharmaceutical sciences
}

\author{
Jan Feijen \\ Department of Chemical Technology and Institute for Biomedical Technology, BMTI, P.O. Box 217, 7500 AE Enschede, Netherlands
}

\section{Introduction}

The design of materials for specific biomedical applications has rapidly expanded during the last 20 years. Biomaterials are generally divided into polymeric materials, ceramics and metals. Ceramics and metals are mainly used for the replacement of hard tissue (orthopaedics), whereas polymeric materials are applied for both the (temporary) replacement of soft and hard tissues. Combinations of different materials are applied in medical devices like catheters, sensors and in artificial organs, (hemodialyzers, oxygenators and artificial hearts). For most applications the surfaces of the materials have to be specifically designed, for instance to prevent thrombus formation when the materials are exposed to blood, to prevent adverse tissue reactions after implantation and to prevent infections when the materials are used for extended periods in the body.

A specific field of interest is the use of polymeric materials or polymers in solution for pharmaceutical applications. Several polymer chemists active in the biomaterials area have become involved in the design of novel systems for the controlled release of drugs. In this lecture new approaches and future perspectives for the use of polymeric materials in drug-delivery systems and of drugs coupled to polymeric surfaces will be discussed.

\section{Tailor-made biodegradable polymers}

Biodegradable polymers have been developed for applications like sutures and the temporary replacement of soft tissues. More recently these polymers have been used in drug-delivery sys- tems especially as biodegradable microspheres. Nowadays the synthesis of different types of biodegradable materials (polyesters, poly $(\alpha$-amino acids), polydepsipeptides, polyorthoesters, polyanhydrides and polyphosphazenes) has rapidly progressed [1-7].

For instance, with respect to the polyesters, (co)polymers with a balance of hydrophilic and hydrophobic properties, stereocomplexes, starbranched polymers and polymers with phaseseparated domains having different affinities for a particular drug can be readily obtained. These polymers have not yet been investigated in detail with respect to the programmed delivery of specific drugs. The rate of degradation of biodegradable polymers can be optimized for a specific application by changing its composition and molecular weight [8]. Detailed studies about the degradation mechanism of degradable polymers have been carried out [9] but there are still aspects which are not completely understood, like the occurrence of autocatalysis and surface to center segregation during degradation. Techniques to combine the polymers with the drug in an adequate dosage form are becoming available. These dosage forms include microspheres, nanoparticles and -spheres, hollow fibers and implants. In the case of nanospheres, novel methods have been developed to modify the surfaces of the spheres in such a way that the spheres are not readily taken up by the reticuloendothelial (RES) system.

An important aspect is the fate of the delivery system after implantation. Toxic, immunologic and carcinogenic reactions have to be avoided. Furthermore, the material-tissue interaction should be at an acceptable level.

The system should be sterilizable and manufacturable in a reproducible way. 


\section{Macromolecular prodrugs targeting}

An exciting approach in the delivery of drugs is to target the drug to the site where it has to exert its function. Very toxic drugs like cytostatic agents can be coupled to a biodegradable or non-biodegradable carrier system, which is provided with a targeting agent (antibody or sugar residue). After coupling, the drug is available in a non-toxic form. The drug has to be coupled to the carrier system in such a way that after arrival at the desired place, in this case in the tumor cell or at the cell wall, it can be released from the carrier by local enzymatic action or by non-enzymatic hydrolyses usually at a different $\mathrm{pH}$ than that in the circulation. For this purpose specific biodegradable spacers have been developed [10].

Problems encountered are that the macromolecular prodrugs can also be taken up by other cells than the target cells. Furthermore, the penetration of the macromolecular prodrug into solid tumors is often not sufficient to establish the required level of the drug.

A recent development is the use of relatively low molecular weight micellar systems, which seem to have a good effectivity for solid tumors [11].

\section{Stimuli-sensitive polymers}

Recently new classes of polymers and polymer networks have become available, in which the swelling properties are dependent on external stimuli, such as $\mathrm{pH}$, temperature, ionic strength and specific chemicals [12]. These polymers and networks usually contain isopropylacrylamide and a variety of charged and hydrophobic comonomers. In principle these systems can be applied to deliver drugs by using a specific stimulus. The mechanism of the stimuli-sensitive swelling and the possible use of these materials will be discussed.

\section{Encapsulation of cells}

Living cells such as islet cells have been encapsulated with different types of polymers [13]. These so-called hybrid systems are being de- veloped to guarantee a glucose-level-dependent release of insulin and to prevent recognition and foreign-body reactions against the cells after implantation.

Current problems are to prepare a stable, uniform coating with the required permeability for glucose and insulin but with a good cut-off for other protein molecules. Furthermore, the encapsulated cells have to be removed from the body in an adequate way after the cells have died.

\section{Surface modification of materials}

When polymeric materials come into contact with blood, clotting usually takes place. There are several methods to prevent clotting and one method is the attachment of the anticoagulent heparin at the surface of the polymeric material. Several investigators have developed methods for the covalent attachment of heparin at polymeric surfaces. Heparin, which has to interact with antithrombin III to exert its activity, has to be attached to the surface in such a way that its activity after coupling is retained. Current methods to couple heparin at surfaces using different kinds of spacers will be discussed and the effectivity of the coatings will be critically evaluated [14].

\section{References}

[1] Jacobs, C., Dubois, Ph., Jerome, R. and Teyssie, Ph. (1991) Macromolecules 24, 3027.

[2] Kricheldorf, H.R. and Meier-Haack, J. (1993) Makromol. Chem. 194, 715.

[3] Van Heeswijk, W.A.R., Hoes, C.J.T., Stoffer, T., Eenink, M.J.D., Potman, W. and Feijen, J. (1985) J. Controlled Release 1, 301.

[4] In't Veld, P.J.A., Dijkstra, P.J., Van Lochem J.H. and Feijen J. (1990) Makromol. Chem. 191, 1813.

[5] Heller, J. (1990) Biomaterials 11, 659.

[6] Domb, A.J. and Langer, R. (1987) J. Polymer Sci: Part A: Polymer Chem. 25, 3373.

[7] Crommen, J., Vandorpe, J. and Schacht, E. (1993) J. Controlled Release 24, 167.

[8] Holland, S.J., Tighe, B.J. and Gould, P.L. (1986) J. Controlled Release 4, 155.

[9] Li, S.M., Garreau, H. and Vert, M. (1990) J. Materials Sci., Materials in Medicine 1, 123; ibid 1, 131.

[10] Kopěcek, J. (1990) J. Controlled Release 11, 279. 
[11] Kwon, G. Suwa, S., Yokoyama, M., Okano, T., Sakurai, Y, and Kataoka, K. (1994) J. Controlled Release 29, 17.

[12] Okano, T., Bae, Y.H. and Kim, S.W. (1990) In: J. Kost (Ed.) Modulated Controlled Release Systems. CRC Press, Boca Raton, FL.
[13] Uludag, H., Babensee, J.E., Roberts, T., Kharlip, L., Horvath, V. and Sefton, M.V. (1993) J. Controlled Release 24, 3.

[14] Vulic, I., Okano, T., Van der Gaag, F.J., Kim, S.W. and Feijen, J. (1993) J. Materials Sci., Materials in Medicine 4, 448. 